\title{
Lithium niobate photonic crystal waveguides: Far field and near field characterisation
}

\author{
M.-P. Bernal*, N. Courjal, J. Amet, M. Roussey, C.H. Hou \\ Institut FEMTO-ST (UMR 6174), Département d’Optique P.M. Duffieux, Université de Franche-Comté, 25030 Besançon Cedex, France
}

\begin{abstract}
In this paper, we experimentally investigate photonic crystal waveguides in a X-cut lithium niobate substrate. The transmission response is measured through the $\Gamma M$ direction of a triangular lattice structure and the results coincide with the theoretical predictions. In addition, a scanning near-field microscope is used in collection mode to map the optical intensity distribution inside the structure putting in evidence the guiding of the light through lines of defects. This study offers perspectives towards lithium niobate tunable photonic crystal devices.
\end{abstract}

Keywords: Lithium niobate photonic crystal; SNOM; Waveguides

Photonic crystals (PCs), also known as photonic bandgap materials, are attractive optical materials for controlling and manipulating the flow of light. Their structure consists basically on periodic changes of the dielectric constant on a length scale comparable to optical wavelengths. Multiple interference between scattered light waves can eventually lead to some frequencies that are not allowed to propagate, giving rise to forbidden and permitted bands, similar to the electronic bandgap in a semiconductor. The band structure depends on the geometry and the material refractive index. Hence, an attractive feature of photonic crystals consists in tuning the substrate refractive index controlling therefore the transmission response. With tunable photonic crystals, the path is open towards high density ultra-compact photonic circuits. This perspective has motivated various studies on tunable photonic devices [1-5].

Among optical tunable materials, the combination of excellent electro-optical, acousto-optical, non-linear optical properties, electro-mechanical (piezoelectric) properties,

\footnotetext{
* Corresponding author. Tel.: +33 3816664 10; fax: +33 381666423 . E-mail address: maria-pilar.bernal@univ-fcomte.fr (M.-P. Bernal).
}

chemical and mechanical stability makes lithium niobate (LN) an attractive host material for application in photonic crystal devices. Moreover, LN high electro-optical coefficient and its low optical losses make it very adequate for optical communication systems.

In our two previous works [6,7], we have shown the fabrication by focused ion beam (FIB) milling of a triangular lattice of nanometric-sized holes with etching depths of $2 \mu \mathrm{m}$ on an annealed proton exchanged (APE) lithium niobate waveguide [7]. We have also shown both theoretically as well as experimentally the presence of a photonic bandgap (PBG) with an extinction ratio lower than $-12 \mathrm{~dB}$.

Recently, an alternative fabrication technique that consists of electric poling and subsequent etching has confirmed the interest in LN-based nanodevices [8].

In this work, the possibility of guiding the light is experimentally evaluated for photonic crystal waveguides. We present a far field as well as a scanning near field experimental characterization of a photonic crystal waveguide fabricated on a lithium niobate waveguide.

As mentioned above, our final objective is the fabrication of photonic bandgap structures in which transmission can 
be tuned by changing the refractive index. We have already theoretically shown [7] that in the case of a triangular array of holes, the optimal sensitivity to the refractive index is obtained when the direction of propagation is $\Gamma M$, and the polarization of the electric field is TE (parallel to the substrate plane and perpendicular to the direction of the holes). The $\Gamma M$ propagation direction exhibits the additional advantage of requiring a lower number of rows to obtain a photonic gap. Indeed, we have shown in Ref. [7] that the $\Gamma M$ direction requires only 15 rows to get a $-12 \mathrm{~dB}$ extinction ratio as opposed to the 30 rows that would be necessary to achieve the same gap in the propagation direction $\Gamma K$. Due to the well-known difficulty to etch lithium niobate, this property has strongly motivated our choice. In addition, with such a configuration we have experimentally demonstrated the existence of a photonic gap.

To complete the analysis of this configuration, we have fabricated two alternative structures, based on the same array as in our previous work, but with one (PCW1) or three lines (PCW3) of defects. The aim is to investigate the possibilities of a tunable guiding of the light through the crystal. The geometrical parameters are chosen to get a transmission zone around $1550 \mathrm{~nm}$ within the bandgap.

The photonic crystals are fabricated on a $0.3 \mathrm{~mm}$ thick Xcut $\mathrm{LiNbO}_{3}$ wafer. In a first step, an optical gradient index waveguide is fabricated by annealed proton exchange. This step is realized through a $\mathrm{SiO}_{2}$ mask in benzoic acid at $180{ }^{\circ} \mathrm{C}$ during $1.5 \mathrm{~h}$. The process is followed by an annealing of the optical waveguide at $333{ }^{\circ} \mathrm{C}$ for $9 \mathrm{~h}$. These parameters are chosen to position the core of the optical mode as close as possible to the surface (approximately $1.4 \mu \mathrm{m}$ ) while keeping single mode propagation at $1.55 \mu \mathrm{m}$.

The photonic crystal structure was fabricated in the central region on the optical channel waveguide as shown in Fig. 1(a). It consists of a triangular lattice of $48 \times 26$ circular holes. The lithium niobate substrate ( 300 microns thickness) is metalised with a thin $\mathrm{Cr}$ layer $(100 \mathrm{~nm})$ to avoid charging effects. This Cr layer is deposited by electron gun evaporation (Balzer, B510). The sample is grounded with a conductive paste before introduction in the FIB vacuum chamber $\left(10^{-6}\right.$ Torr). The FIB used is a FEI Dual Beam Strata 235. $\mathrm{Ga}^{+}$ions are emitted with an accelerated voltage of $30 \mathrm{keV}$ and focused down with electrostatic lenses on the sample with a probe current of $120 \mathrm{pA}$. The Gaussian beam shape spot size is about $20 \mathrm{~nm}$ at the sample surface. The etching time of the structures PCW 1 and PCW3 $(48 \times 26$ triangular hole lattice, hole diameter $=255 \mathrm{~nm}$, periodicity $=510 \mathrm{~nm}$, etching depth $=1500 \mathrm{~nm}$ ) was $20 \mathrm{~min}$ each. We would like to point out that the removal of material by FIB milling is achieved without the use of a patterned resist mask and therefore, high-precision complex structures can be directly fabricated. A FIB image cross-section of the holes is shown in Fig. 1(b). The angle between the FIB beam and the holes axis is $52^{\circ}$.

In order to couple the conventional TE APE mode ( $4 \mu \mathrm{m}$ size) to the photonic crystal waveguides (approxi-

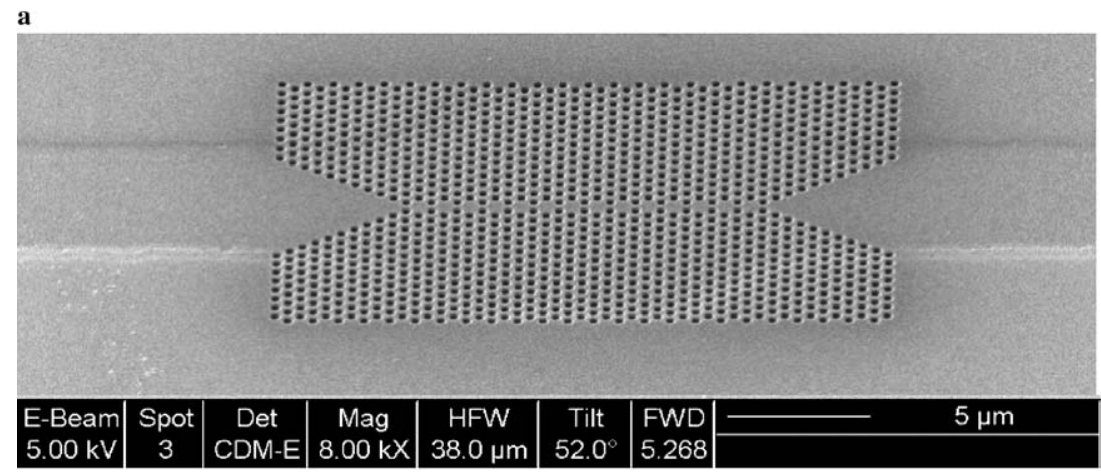

b

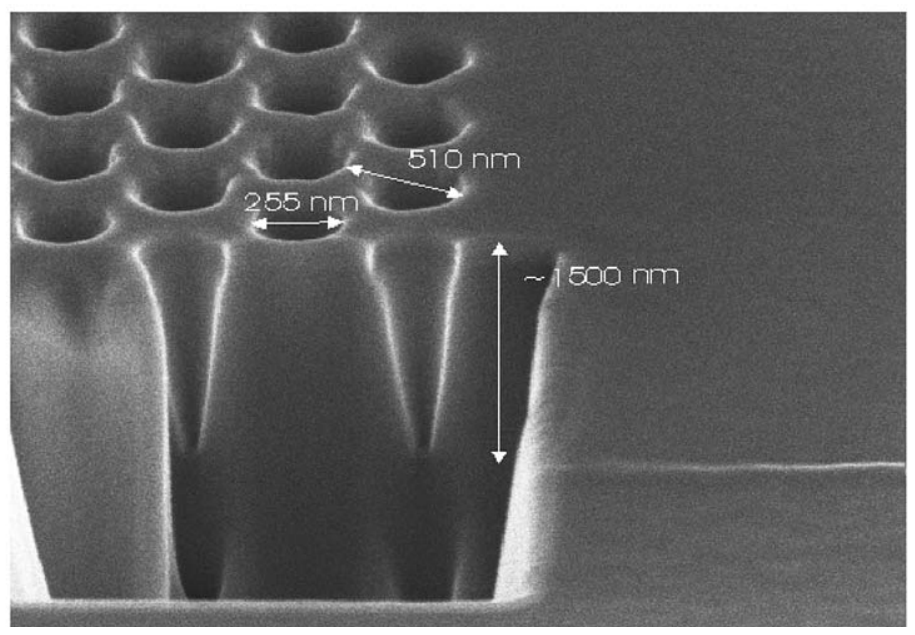

Fig. 1. SEM view of the: (a) triangular lattice PCW1 and (b) its cross-section. 

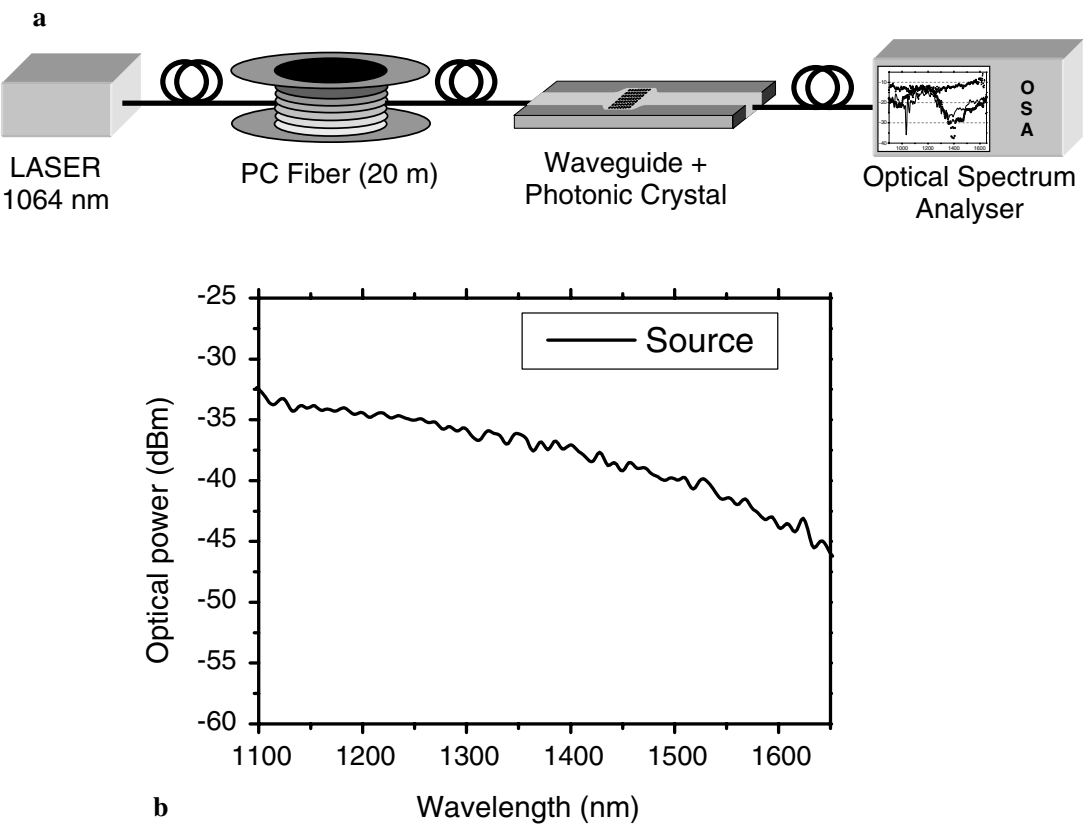

Fig. 2. (a) Experimental setup and (b) super-continuum at the output of the PC fibre.

mately $500 \mathrm{~nm}$ and $1500 \mathrm{~nm}$ wide), the optical mode is smoothly guided through a photonic tapered structure (see Fig. 1(a)).

The novel structures were first characterized by measuring their far field transmission. The experimental setup is shown in Fig. 2(a). In order to get a spectrum as flat as possible on a large range of wavelengths $(1000-1700 \mathrm{~nm})$, we use a super-continuum light source. The white light is generated by a sub-nanosecond microchip laser emitting at $1064 \mathrm{~nm}$ with $8 \mu \mathrm{J}$ energy per pulse [9]. The laser light is coupled into a photonic crystal (PC) fibre, which enhances the nonlinear effects required for the generation of a large super-continuum. The resulting output spectrum for a $20 \mathrm{~m}$ long PC fibre is shown in Fig. 2(b).

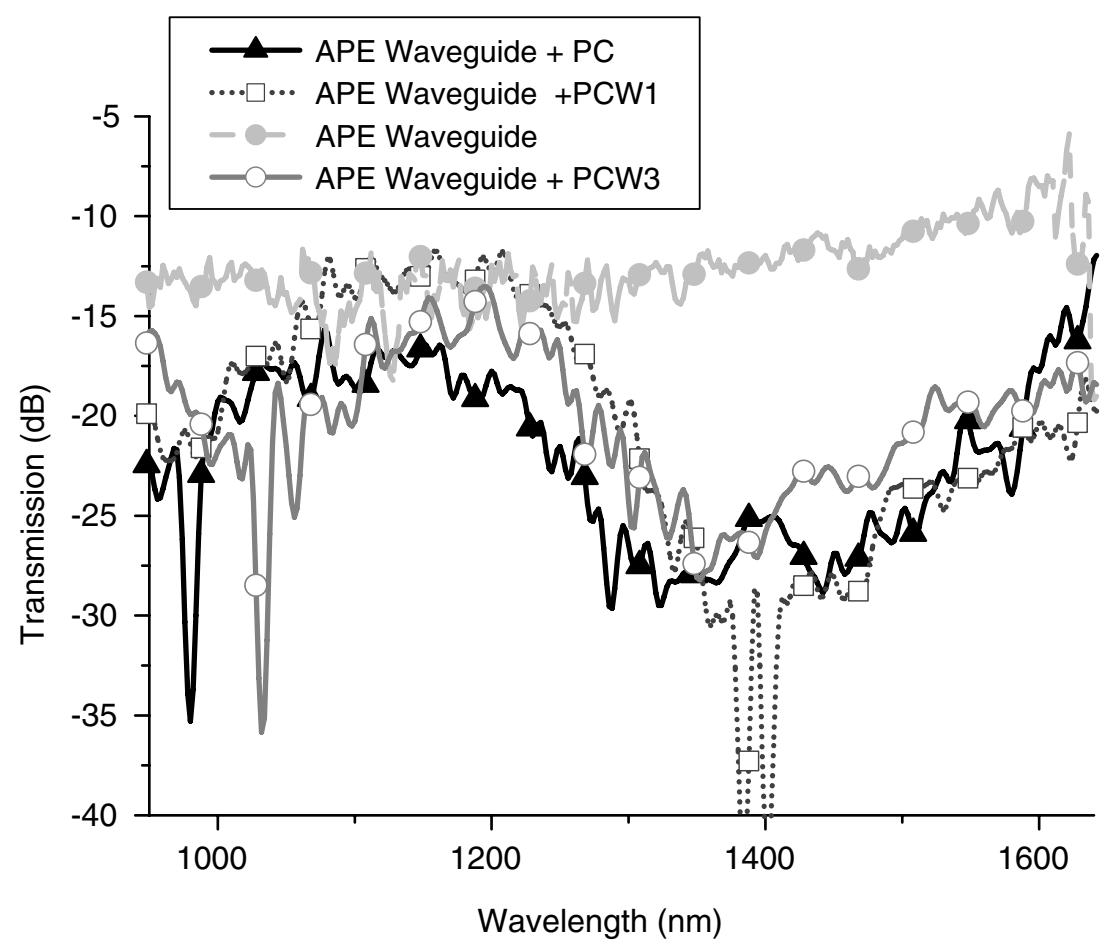

Fig. 3. Experimental transmission through three optical waveguides integrated on the same wafer: Light grey line with solid circle: transmission through an annealed proton exchanged (APE) waveguide. Grey line with empty circles: transmission through a PCW3 integrated on an APE waveguide. Dark grey with empty squares: transmission through a PCW1 integrated on an APE waveguide. Black line with solid triangles: transmission through a PC integrated on an APE waveguide. 
The optical transmission was measured through the two photonic crystal waveguides, a photonic crystal without defect lines, and through a standard optical waveguide, fabricated on the same wafer and in the same conditions as described above. The experimental results are shown in Fig. 3. As it can be seen in the graph, the transmissions through the photonic structures (filled triangle, empty square, empty circle) exhibit a gap, which does not appear
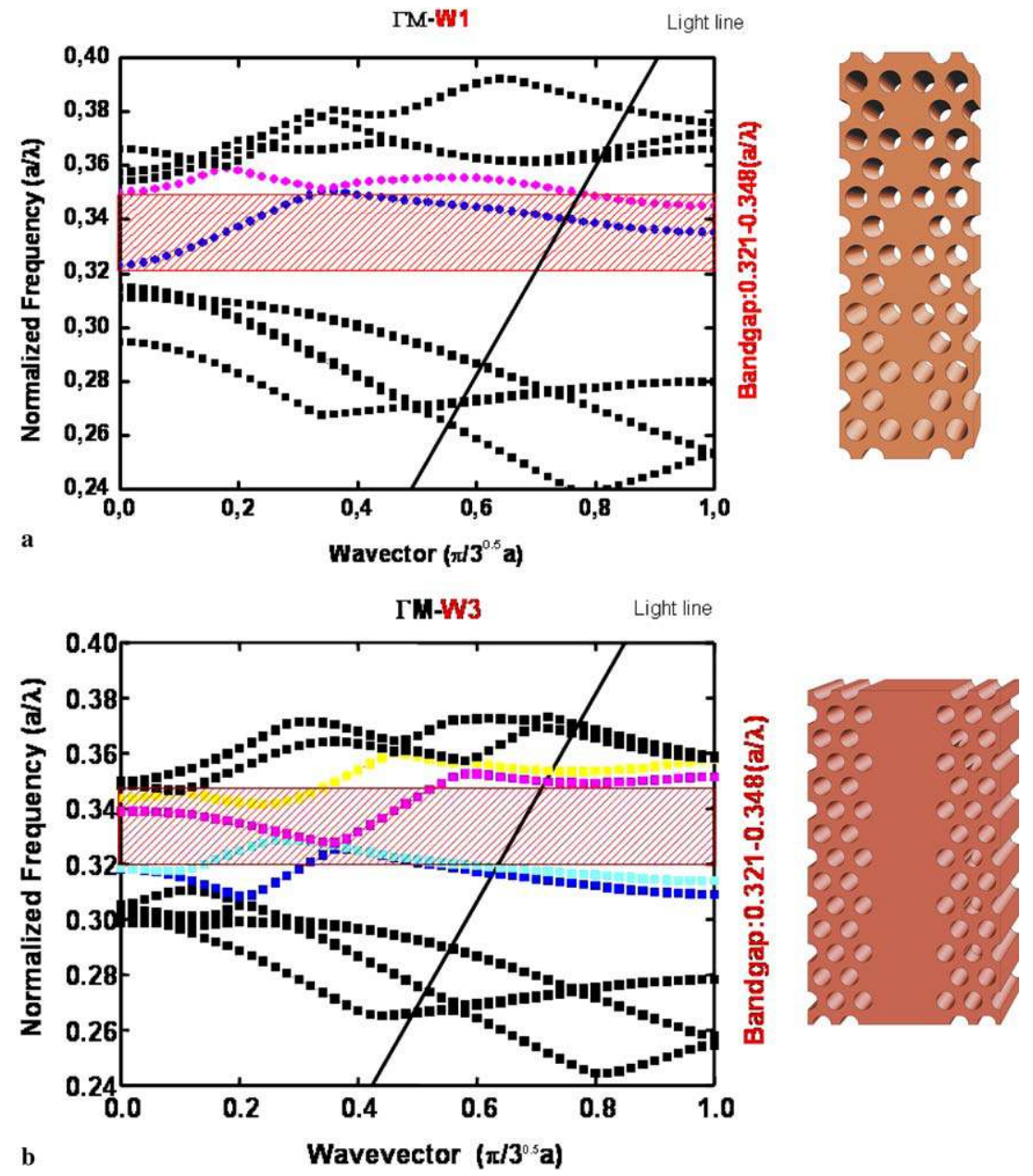

Fig. 4. Projected band diagrams and light line along the $\Gamma M$ direction for the two photonic crystal waveguides: (a) PCW1 and (b) PCW3.

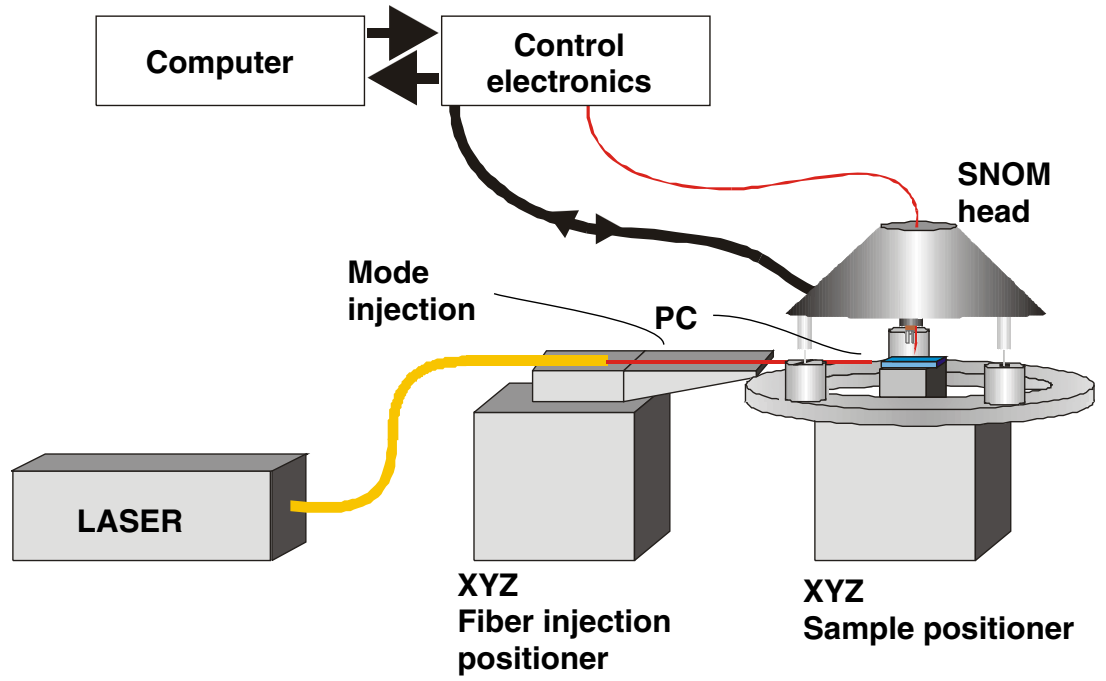

Fig. 5. Experimental set-up of the scanning near-field optical microscope (working in collection mode). 
in the transmission through the single APE waveguide (filled circle). In parallel, 2D-numerical simulations performed with a commercial software (BandSOLVE) of the device without defect lines predict a band gap between $1465 \mathrm{~nm}$ and $1589 \mathrm{~nm}$. The experimental gap starts in a shorter wavelength (approximately around $1300 \mathrm{~nm}$ ) which we believe is a consequence of fabrication imperfections and to the fact that $2 \mathrm{D}$ calculated bandgaps are usually shifted towards shorter wavelengths compared to full 3D simulations. Fig. 4(a) and (b) show the projected band diagrams and the light line for the two photonic crystal waveguides. For the PCW1 case, the diagram shows two guided modes. In the PCW3 case all the modes are radiative (Fig. 4(b)). Experimentally (Fig. 3), light propagation in the PC waveguides is observed by an increase in the transmission inside the gap. This increase is twice more important for the PCW3 case which may be due to a better coupling efficiency of the input taper. We have repeated the measurements five times, and changed the injection conditions in order to verify the position of the gap and to optimize the propagation through the photonic crystal waveguides. The position of the gap did not change in all the five measurements.

For a deeper interpretation of the propagation of the light through the structures, we have also investigated the near field behaviour of the light inside the PC waveguides. The wave fronts of light in the photonic crystal waveguide undergo substantial modulations on length scales that are much shorter than one wavelength. Therefore, it is impossible to resolve the spatial details of light propagation by the far field transmission measurement described above. Although still not systematically used in the photonic crystal community, several groups have shown already very interesting results in near field characterisation of photonic crystals [10-17].

In the work presented here, the instrument used is a commercial scanning near-field optical microscope (SNOM) (NT-MDT SMENA) in collection mode [18]. The near-field optical fibre probe is fabricated by heating a single mode optical fibre with a $\mathrm{CO}_{2}$ laser and then pulling it apart with a micropipette puller (Sutter Instrument Co.) to obtain a sharp taper region with a small end face $(\sim 100 \mathrm{~nm})$. To obtain the SNOM images, one needs to scatter the evanescent fields on the sample by raster scanning the sub-wavelength probe at a few nanometers from the surface. A non-optical shear force feedback [19] is used to keep the probe at a constant distance from the sample surface. Both signals, the feedback and the optical one, are simultaneously acquired to construct topographic and SNOM collection images.

Fig. 5 shows the experimental set-up. To image the transmitted mode through the LN photonic structure, two different laser sources and two different optical detectors have been utilized. The first acquisition has been per-
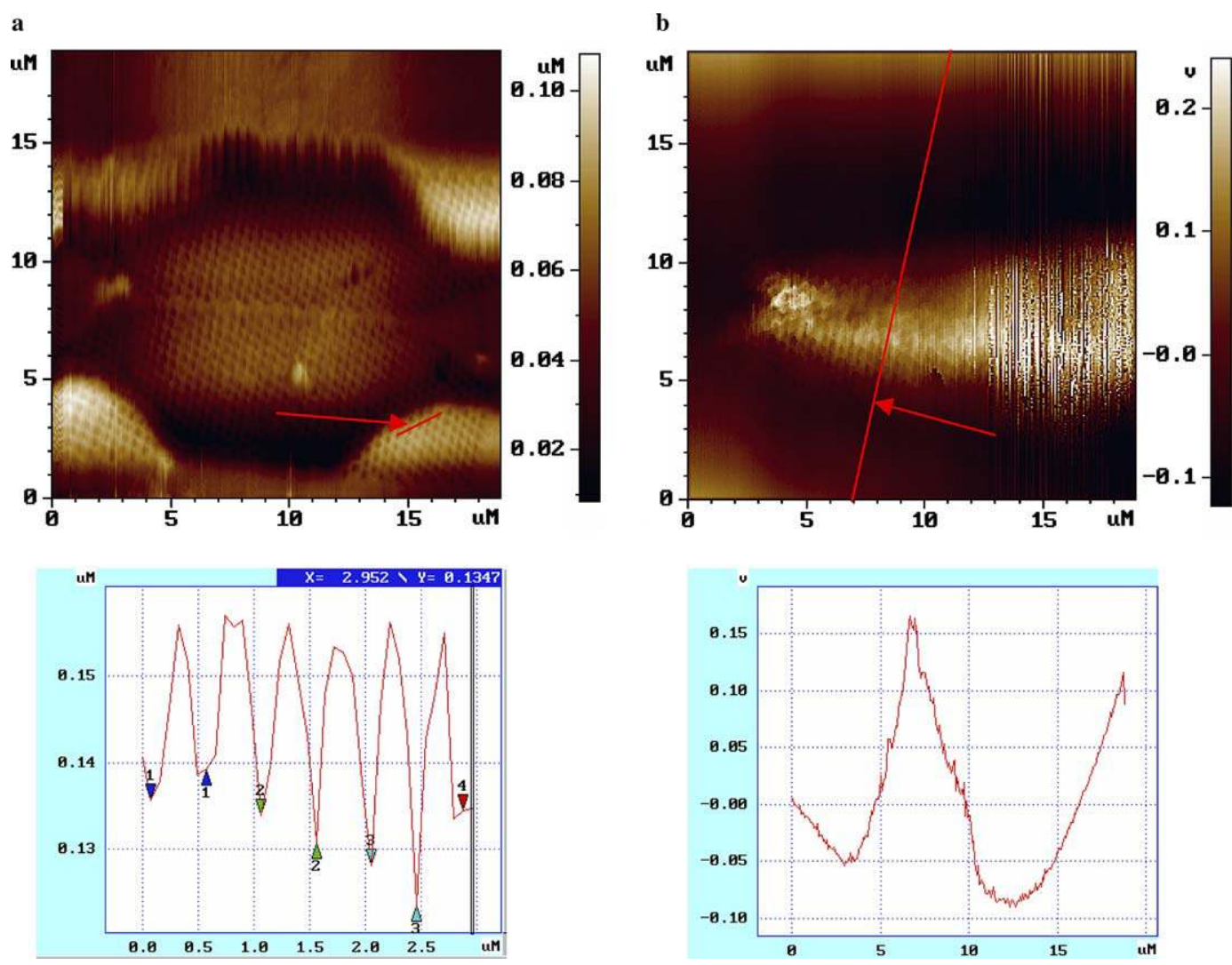

Fig. 6. (a) Topographical image of $20 \times 20 \mu \mathrm{m}$ size of the PCW1 structure. The inset shows a cross-section through 7 holes inside the structure. (b) The simultaneously recorded optical near field of the structure when the coupled wavelength is $810 \mathrm{~nm}$. The inset shows the width of the optical field inside the structure. 
a
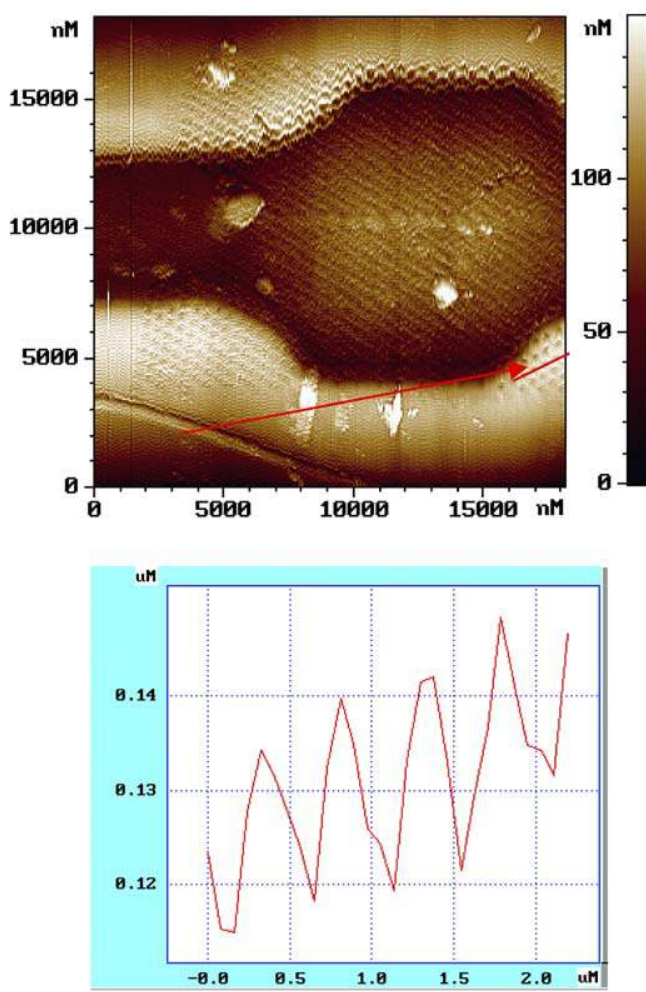

b
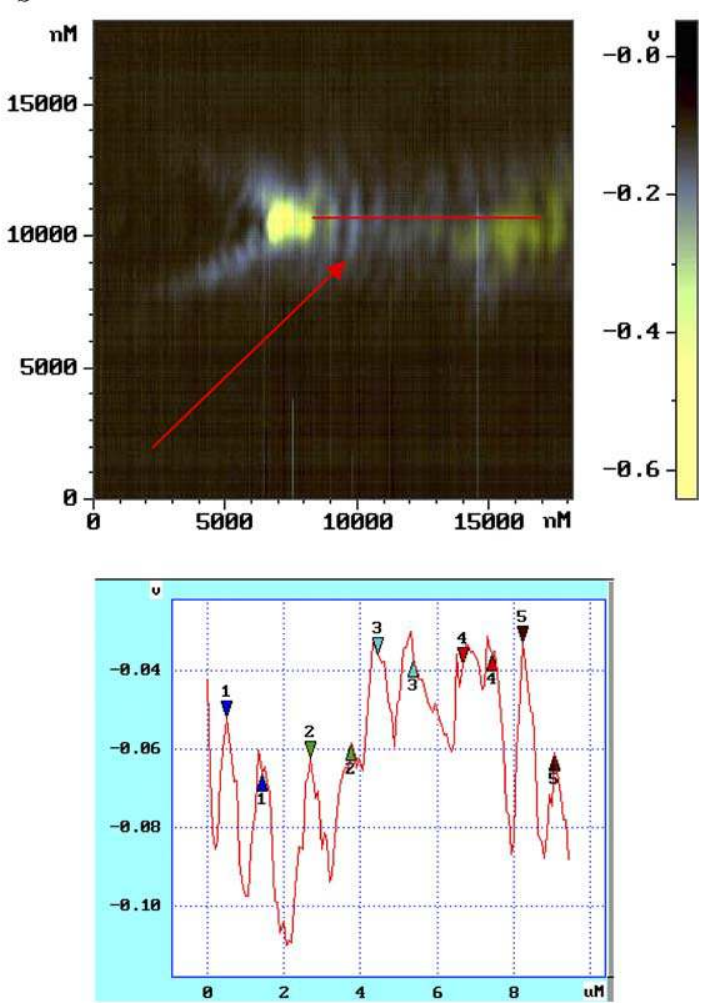

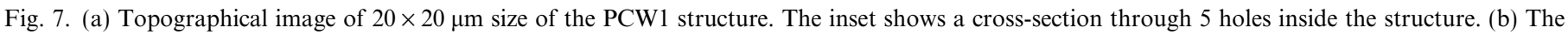

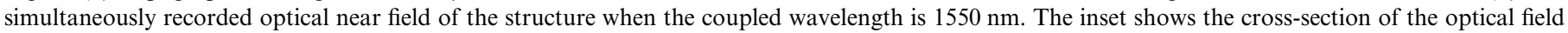
along the propagation axes.

formed at $810 \mathrm{~nm}$ (outside the gap), with a Thorlabs STFC780 laser and detected with an Oriel 70680 photomultiplier. The second acquisition has been realized at $1.55 \mu \mathrm{m}$ with a distributed feedback laser OKI OL502OON and an InGaAs detector (Thorlabs D400FC), to characterize the propagating region inside the gap.

The optical image and topography of the PCW1 at $810 \mathrm{~nm}$ is shown in Fig. 6. Fig. 6(a) shows the topography of the PCW1 structure and a zoom of a small region that consists of 7 holes. The hole depth measured by the SNOM tip is of the order of $30 \mathrm{~nm}$ which is far from the $1.5 \mu \mathrm{m}$ measured in the SEM image. This is basically due to the fact that the hole diameter is comparable in size to the tapered fibre, being difficult for the tip to penetrate inside the holes. The signal to noise ratio (SNR) is however high ( 10). Fig. 6(b) shows the optical image of the light going through the PCW1 at $810 \mathrm{~nm}$.

We have also performed the near field measurements in a region inside the gap in which an optical mode propagates $(\lambda \sim 1.55 \mu \mathrm{m}$, transmission $\sim-25 \mathrm{~dB})$. The results are shown in Fig. 7. Fig. 7(a) shows the topography and Fig. 7(b) the near field image respectively. It is worth mentioning that this set of measurements have been performed with a different SNOM probe than for the case of $810 \mathrm{~nm}$ due to the tip destruction. Again, the topography shows clearly the photonic structure with a SNR of 10 . We can very well appreciate the tapered beginning and end of the structure and the line of defects. The simultaneously recorded optical signal is shown in Fig. 7(b). This image corresponds to an input wavelength of $1.55 \mu \mathrm{m}$. The recorded signal shows a periodicity of about $800 \mathrm{~nm}$ (see inset in Fig. 7(b)) that corresponds to a Bloch periodicity of $\sqrt{3}$ a ( $\Gamma M$ direction, triangular lattice). With these results, we can infer that the step seen in the transmission response of the PCW1 (Fig. 3) around $1500 \mathrm{~nm}$ is due to the existence of a guiding region.

In conclusion, we have shown the first photonic crystal waveguides fabricated by FIB etching in lithium niobate. In spite of the optical losses that are mainly due to multimode guiding of the standard APE waveguide and the fabrication imperfections caused by the well-known difficulty of etching lithium niobate, a guided mode is successfully observed in the PCW1 structure by near field characterization.

Work is in progress to show experimentally a tunable lithium photonic device with an optimised photonic crystal configuration.

\section{Acknowledgements}

This work has been supported by the Action Concertée Incitative "Nanosciences" COBIAN, No. NR137. The authors thank Dr. Elöise Devaux, ISIS Strasbourg, France for the FIB milling. 


\section{References}

[1] H.M.H. Chong, R.M. De La Rue, IEEE Photon. Technol. Lett. 16 (2004) 1528.

[2] N.C. Panoiu, M. Bahl, R.M. Osgood, Opt. Lett. 28 (2003) 2503.

[3] B. Wild, R. Ferrini, R. Houdré, M. Mulot, S. Anand, C.J.M. Smith, Appl. Phys. Lett. 84 (2004) 846.

[4] M.J. Escuti, J. Qi, G.P. Crawford, Appl. Phys. Lett. 83 (2003) 1331.

[5] B. Li, J. Zhou, L. Li, X.J. Wang, X.H. Liu, J. Zi, Appl. Phys. Lett. 83 (2003) 4704.

[6] F. Lacour, N. Courjal, M.-P. Bernal, A. Sabac, C. Bainier, M. Spajer, Opt. Mater. 27 (2005) 1421

[7] M. Roussey, M.-P. Bernal, N. Courjal, F.I. Baida, Appl. Phys. Lett. 87 (2005) 241101.

[8] S. Grilli, P. Ferraro, P. De Natale, B. Tribilli, M. Vassalli, Appl. Phys. Lett. 87 (2005) 233106.

[9] K.P. Hansen, R.E. Kristiansen, Supercontinuum generation in photonic crystal fibers, Application Note, Crystal Fibre (Available from: <www.crystal-fibre.com>).
[10] E. Flück, M. Hammer, A.M. Otter, J.P. Korterik, L. Kuipers, N.F. van Hulst, J. Lightwave Technol. 21 (2003) 1384.

[11] S.I. Bozhevolnyi, V.S. Volkov, T. Sondergaard, A. Boltasseva, P.I. Borel, M. Kristensen, Phys. Rev. B 66 (2002) 235204.

[12] H. Gersen, T.J. Karle, R.J.P. Engelen, W. Bogaerts, J.K. Korterik, N.F. van Hulst, T.F. Krauss, L. Kuipers, Phys. Rev. Lett. 94 (2005) 073903.

[13] D. Gérard, L. Berguiga, F. de Fornel, L. Salomon, C. Seassal, X. Letartre, P. Rojo-Romeo, P. Viktorovitch, Opt. Lett. 27 (2002) 173.

[14] E. Flück, M. Hammer, W.L. Vos, N.F. van Hulst, L. Kuipers, Photon. Nanostruct. - Fundam. Appl. 2 (2004) 127.

[15] P.L. Phillips, J.C. Knight, B.J. Mangan, P.St.J. Russell, M.D.B. Charlton, G.J. Parker, J. Appl. Phys. 85 (1999) 6337.

[16] P. Kramper, M. Kafesaki, C.M. Soukoulis, A. Birner, F. Müller, U. Gösele, R.B. Wehrspohn, J. Mlynek, V. Sandoghdar, Opt. Lett. 29 (2004) 174.

[17] B. Cluzel, D. Gérard, E. Picard, T. Charvolin, V. Calvo, E. Hadji, F. de Fornel, Appl. Phys. Lett. 85 (2004) 2682.

[18] D.W. Pohl, Optical near-field scanning microscope, United States Patent 4604520.

[19] K. Karrai, R.D. Grober, Appl. Phys. Lett. 66 (1995) 1842. 